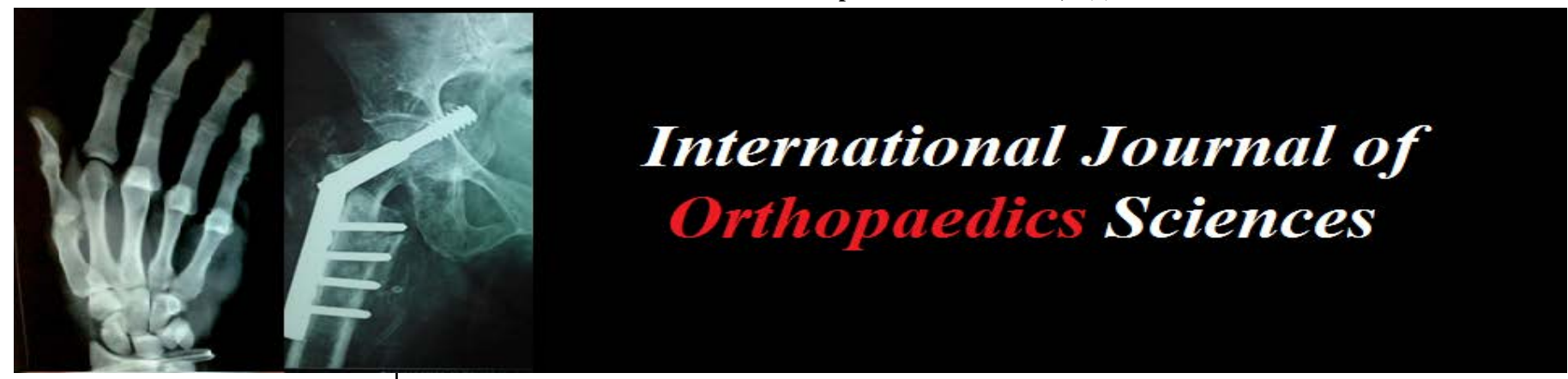

E-ISSN: 2395-1958

P-ISSN: 2706-6630

IJOS 2021; 7(4): 114-118

(C) 2021 IJOS

www.orthopaper.com

Received: 19-08-2021

Accepted: 21-09-2021

Dr. N Vishnu Vardhan

Associate Professor,

Department of Orthopaedics,

Narayana Medical College and

Hospital, Chintareddypalem,

Nellore, Andhra Pradesh, India

Dr. Kuruba Usha Rani

Postgraduate Resident,

Department of Orthopaedics

Narayana Medical College and

Hospital, Chintareddypalem,

Nellore, Andhra Pradesh, India

\section{Management of distal ulna giant cell tumor by en bloc resection and reconstructed by a novel technique}

\author{
Dr. N Vishnu Vardhan and Dr. Kuruba Usha Rani
}

DOI: https://doi.org/10.22271/ortho.2021.v7.i4b.2873

\begin{abstract}
Giant cell tumor (GCT) is a locally aggressive benign primary bone tumor accounting for $20 \%$ of all the benign primary bone tumors. Peak incidence is between 20 and 45 years with slight female predominance. Distal end of ulna is a rare site for any primary bone tumor, especially 2-4 for giant cell tumors with reported incidence of 0.45 to $3.2 \%$ in literature. Considering local aggressive nature and high recurrence, resection is the treatment recommended. In such cases, instability of the ulnar stump and ulnar translation of the carpals are known 3-6 complications causing reduced grip strength. Literature review states, reconstruction with iliac bone graft and stabilization of the ulnar stump with tenodesis using extensor carpi ulnaris. We report a 34 year old female with giant cell tumor of the distal ulna treated by en bloc resection and reconstruction of the distal radio-ulnar joint (DRUJ) and triangular fibrocartilage (TFCC) complex with proximal fibula, palmaris longus graft with augmentation by extensor carpi ulnaris tenodesis and stabilization of the proximal stump with dynamic compression plate. This newer technique of DRUJ and TFCC reconstruction with proximal fibula and palmaris longus graft following en bloc resection of the distal ulna had excellent functional results with no evidence of recurrence after seven months follow-up. This case is presented for its rarity and novelty of the technique used for the anatomical reconstruction.
\end{abstract}

Keywords: Giant cell tumor, distal ulna, en bloc resection, proximal fibular graft, palmaris longus graft

\section{Introduction}

Giant cell tumor (GCT) or Osteoclastoma is a rare, benign tumor with local aggressive nature. It accounts for $4-5 \%$ of all primary bone tumors, commonly affecting distal femur, a proximal tibia, distal radius and proximal humerus. Pulmonary metastases are seen in $2 \%$ of patients with GCT. Local recurrence rate is approximately $25 \%$ with various types of intraluminal ablation procedures described for the GCT. En bloc resection of the GCT is the recommended treatment option with fewer chances of recurrence at this location. We report a patient with GCT of the distal ulna, treated by en bloc resection, followed by reconstruction of the distal ulna and triangular fibrocartilage complex (TFCC) using proximal fibula, palmaris longus tendon and a part of extensor carpi ulnaris with stabilization of the proximal stump using dynamic compression plate. To the best of our knowledge, this is a novel technique to be reported.

\section{Case report}

A 34-year-old South Indian female, presented with a single painless swelling of left distal ulna of one and half years duration. Initially the ulna head was more prominent compared to the opposite side. It gradually increased to present size. There was no history of fever, loss of appetite or weight and there was no other significant history. On examination, the swelling was oval in shape measuring $5.0 \times 4.0 \mathrm{~cm}$, occupying the distal third ulna (Fig.1 \& 2) Skin over the swelling was normal in colour and temperature. The swelling was diffusely tender, firm in consistency and was fixed to the underlying bone. But skin over the swelling was pinchable. The range of movements of the wrist was full and painless. Hand grip was normal in both hands with normal distal neurovascular status. There was no lymphadenopathy or any other systemic disease. Hematological and biochemical parameters were within normal limits. Plain chest radiograph was normal.
Corresponding Author: Associate Professor, Department of Orthopaedics, Narayana Medical College and Hospital, Chintareddypalem, Nellore, Andhra Pradesh, India 
Plain radiograph of the left wrist in both AP and lateral views showed single, expansile, multiloculated lytic lesion involving the epiphysis and metaphysis of distal end of ulna with no cortical breach or periosteal reaction (Fig.3). It extends upto the subchondral bone of articular cartilage with no intralesional calcifications. CT scan showed expansile lytic lesion with cortical thinning and areas of cortical destruction. (Fig.4) MRI both plain \& contrast showed $6.5 \times 5.6 \times 5 \mathrm{~cm}$, isointense $\mathrm{T} 1 \mathrm{~W}$ and hyperintense $\mathrm{T} 2 \mathrm{~W}$ lesion in the distal ulna (Fig.5,a \& b ) involving the epiphyseal-metaphyseal region which enhances with contrast. Cortical break was noted with TFCC involvement. Also edema of the adjacent tissues was present. Both clinically and radiologically the features were suggestive of giant cell tumor of the distal end of ulna.
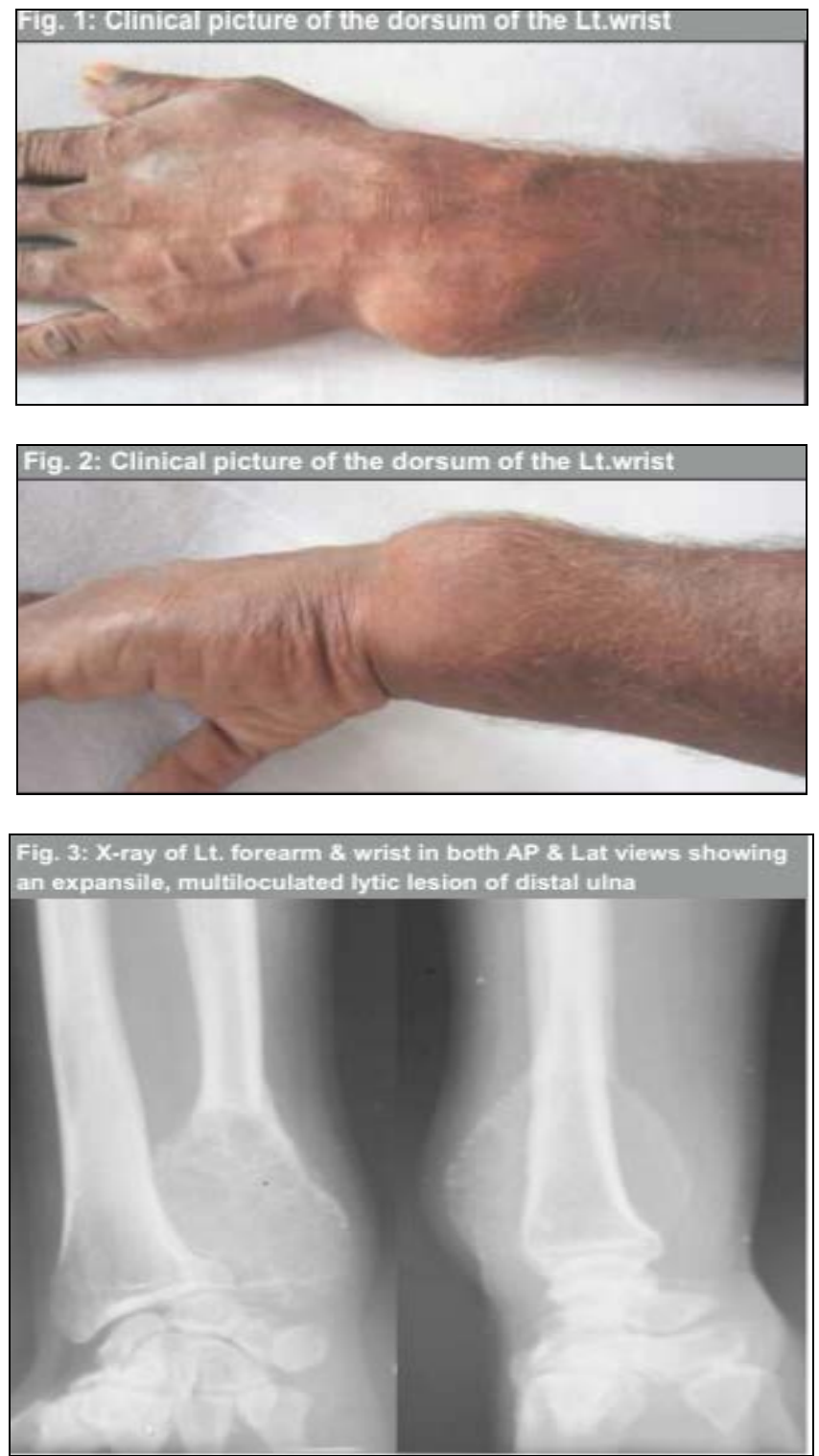
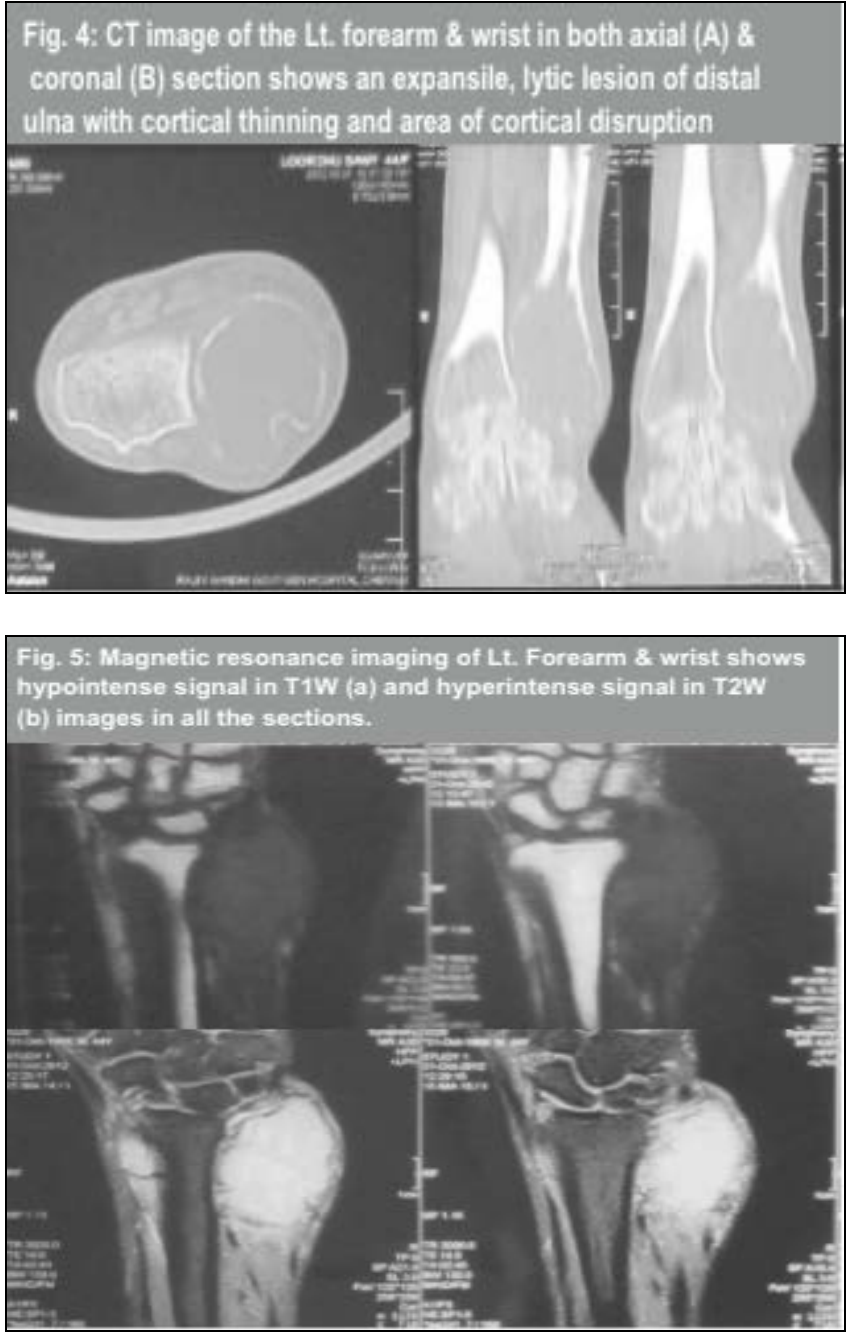

Fine needle aspiration cytology showed a double cell population consisting of stromal cell clusters and numerous osteoclastic giant cells. This confirmed our diagnosis as a giant cell tumor of distal ulna. Based on the clinical and radiological evaluation, the tumor was graded as aggressive type (grade 3) as per the enneking classification of giant cell tumors of bone. Hence, as per the recommended treatment, en bloc resection was planned. The disease, prognosis with chances for metastasis or recurrence and treatment advised were all discussed with the patient in detail and informed written consent was obtained.

The patient was taken up for extra periosteal en bloc resection of the tumor. Through a $10 \mathrm{~cm}$ incision over the dorsal and medial aspect of the left distal ulna, the tumor was exposed completely. The extensor carpi ulnaris (ECU) tendon was found almost engulfed by the tumor mass (Fig.6a). Sparing the ECU, the tumor was resected en bloc with $2 \mathrm{~cm}$ cuff of normal bone (Fig.6b). The excised specimen was $8 \mathrm{~cm}(6+2$ $\mathrm{cm}$ ) in length and $4 \mathrm{~cm}$ in breadth (Fig.6c).

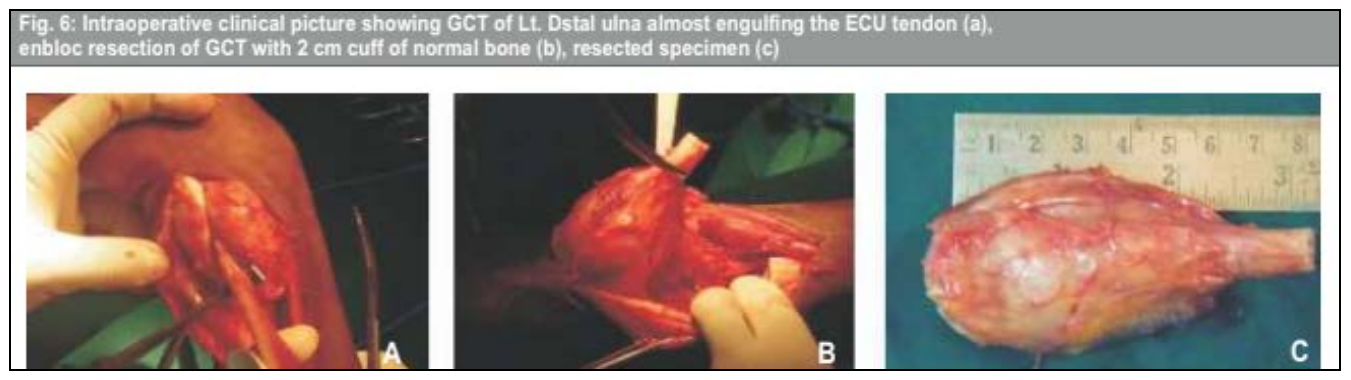


Considering the age of the patient and high level of functional demands, distal ulna with distal radio-ulnar joint (DRUJ) and triangular fibrocartilage complex (TFCC) reconstruction was planned. Then a proximal fibula of $9 \mathrm{~cm}$ was harvested and the fibular head was resized to match the size of the ulnar head (Fig.7a). After preparation, the proximal fibular graft was kept in the defect, with resized fibular head in place of ulnar head. Precaution was taken to prevent the raw area of the resized fibula from facing the radius, this is to prevent cross union at DRUJ. Palmaris longus tendon was harvested and used as a tendon graft for the reconstruction of the TFCC and DRUJ as described by Adams and Berger in the treatment of chronic DRUJ instability (Fig.7b). This construct was stabilized with two parallel k-wires across the fibular graft and distal radius (Fig.7c). Also ECU tenodesis was done to augment the construct (Fig.8a \& 8b).
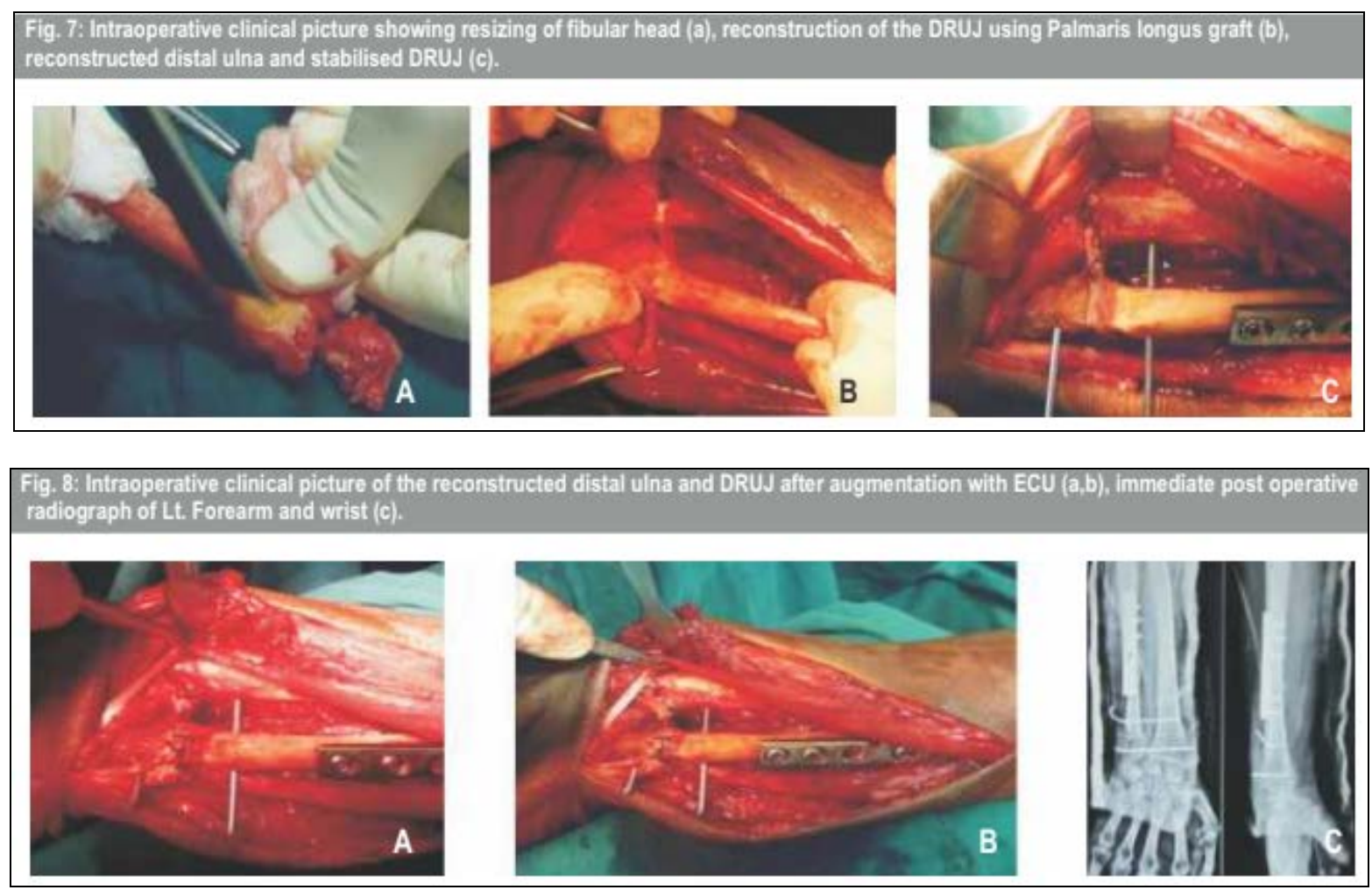

Proximally the fibular graft was stabilized with the proximal ulnar stump using a 6 holed dynamic compression plate. The k-wires were kept outside the skin for easy and earlier removal of the same. Wound was closed in layers with drain left in situ. Left upper limb was immobilized in above elbow (AE) cast with elbow in 90 deg flexion and forearm in full supination (Fig.8c). Wound inspection and suture removal was done through a window made in the cast. After wound healing, AE cast with elbow in 90 deg flexion and forearm in full supination was reinforced and continued till 6 weeks. Kwires were removed at 6 weeks. After that, gentle elbow, forearm, wrist and hand mobilization exercises were started. 


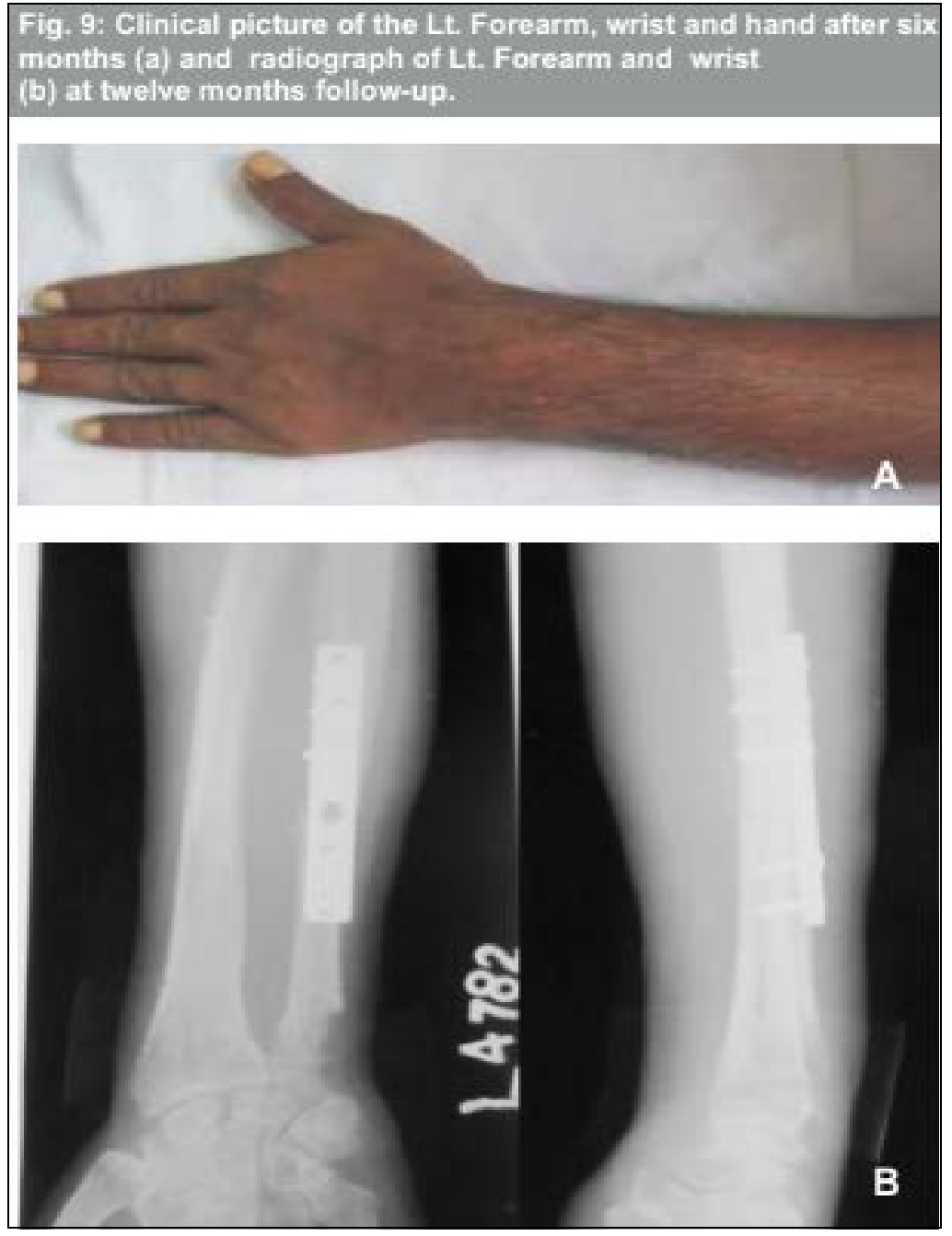

Follow-up was advised at 3 months, 6 months and once in six months for two years. After one year, sound union of the graft was noted with good functional range of movements at wrist and forearm with excellent hand grip and no evidence of carpal instability or recurrence of the tumor.

\section{Discussion}

Primary GCT of distal ulna is rare, with reported annual 2, 3, 4 incidence of 0.45 to $3.2 \%$. Various treatment options are available based on the clinical and radiological staging of tumors by Enneking. Stage I or Latent lesions require no active treatment. Stage II or Active lesions require intralesional curettage with adjuvant therapy. Stage III or Aggressive lesions require en bloc resection. Cooney et al, achieved excellent results in $75 \%$ of their eight cases, which required wide excision of the distal ulna for various tumors. He concluded that routine reconstruction of the osseous defect is not needed for all cases. On the other hand, many authors have documented, poor functional results following en bloc resection of the distal ulna for those reasons like pain and restriction of movements due to impingement of the ulnar stump on the radial metaphysis and dorsal winging of the ulnar stump or radio-carpal instability with ulnar translation of the carpus. The distal ulna was initially considered as a dispensable bone and distal ulnar resection was recommended for degenerative conditions as Darrach's procedure. Though distal ulnar resection following any tumor is not comparable to Darrach's procedure for various reasons the later procedure too has unpredictable long-term results. Hence, there arises the need for anatomical reconstruction of the distal ulna and the distal radio-ulnar joint with stabilization of the proximal ulnar stump for better functional results especially in young patients with high functional demands. Various authors suggested different techniques for reconstruction or stabilization after en bloc resection of distal ulna for primary neoplasms. These include stabilization by tenodesis, auto graft or allograft replacement, plate arthrodesis and bone transport using ilizarov ring fixator. Gainer reported lasso tenodesis of the ulnar stump with tendon graft from Palmaris longus, in two cases of GCT after 15 wide excision. Ferracini et al reported five patients with GCT and 3 patients with other tumors involving distal ulna. They stabilized ulnar stump using flexor carpi ulnaris (FCU), fascia lata and autograft. They suggested that soft tissue stabilization of the ulnar stump should be done whenever possible. Goldner and Hayes in 1979, was the first to describe ECU tenodesis to stabilize the ulnar stump based on their experience on 225 patients, who underwent distal ulna excision for various conditions. Following them, many the authors reported this procedure after wide excision of the distal ulna. Wurapa and Whipple reported a two stage allograft reconstruction of the DRUJ. With 40 months follow-up. patient had extremely satisfactory results.

Stofflen et al. reported the use of ilizarov fixator for the transport of proximal ulnar stumps. Patient had an acceptable and painless range of movements of the wrist. Hashizume et al. described an ulnar buttress arthroplasty following en bloc resection of the distal ulna for the first time. They used a longer iliac bone graft on the ulnar side of the radius using a screw and k-wire to attain the buttress against axial loading. They reported excellent results with six months follow-up. Similarly, Minami et al. Reported satisfactory results by 
following a technique of modified ulnar arthroplasty in a young adult after resection of GCT of the distal ulna. Monappa A Naik et al. reported excellent functional outcome with their technique of ulnar buttress arthroplasty, almost similar to minami et al report, following en bloc resection of GCT distal ulna in a 34 year old female. From the literature review, we can understand that, reconstruction of DRUJ along with stabilization of the ulnar stump is mandatory in any functionally demanding individual, following en bloc resection of the distal ulna. In our technique, instead of iliac crest graft, proximal fibula is used for the reason of more anatomical similarity to the distal ulna. We had replaced the fibular graft, for the entire length of the ulna resected. Proximally a six holed narrow DCP was used to stabilize the fibular graft with the ulnar stump. Also in our technique, DRUJ \& TFCC was reconstructed with Palmaris longus graft using the technique, similar to the one described by Adams and Berger for DRUJ reconstruction in chronic 20 DRUJ instability. Hence, there is no question of either ulnar stump instability or radio-carpal instability with translation of the carpals in our technique. In literature, ECU tenodesis was done only to stabilize the proximal ulnar stump. But In our technique, ECU tenodesis was done for the augmentation of the DRUJ and not for the stabilization of the proximal ulnar stump.

\section{Conclusion}

Our case is to be reported, using this entirely novel technique of reconstruction of the entire length of excised distal ulna, DRUJ and TFCC using proximal fibula, Palmaris tendon graft, ECU, K-wires and dynamic compression plate for GCT of distal ulna in a 34-years-old functionally demanding female with excellent functional results in range of movements, stability and good hand grip with no evidence of recurrence after one year follow-up.

\section{References}

1. Christopher Fletcher DM, Krishnan Unni K, Fredrik Marten's. Pathology and Genetics of Tumours of Soft Tissue and Bone. WHO Classification of tumors 2002, 338-9.

2. Goldenberg RR, Campbell CJ, Bonfiglio M. Giant cell tumor of bone. An analysis of two hundred and eighteen cases Joun Bone and Joint Surg 1970;524:619-64.

3. Kayias EH, Drosos GI, Anagnostopoulou GA. Resection of the distal ulna for tumours and stabilization of the stump. A case report and literature review. Acta Orthop Belg 2006;72:484-91.

4. Naik MA, Sujir P, Rao SK, Tripathy SK. Ulnar buttress arthroplasty after en bloc resection of a giant cell tumor of the distal ulna. Indian Journal of Orthopedics 2013;47(2):211-14.

5. Singh M, Sharma S, Peshin C, Wani IH, Tikoo A, Gupta SK, et al. Wide resection and stabilization of ulnar stump by extensor carpi ulnaris for giant cell tumor of distal ulna: two case reports. Cases J 2009;2:8617.

6. Minami A, Iwasaki N, Nishida K, Motomiya M, Yamada K, Momma D. Giant-cell tumor of the distal ulna treated by wide resection and ulnar support reconstruction: A Case report. Case reports in Medicine ID 871278 2010, 4.

7. Enneking WF. A System of staging musculoskeletal neoplasms. Clin Orthop Relat Res 1986;204:9-24.

8. Ferracini R, Masterson EL, Bell RS, Wunder JS. Distal ulnar tumours. Results of management by en bloc resection in nine patients and review of the literature. $\mathrm{J}$
Hand Surg [Br] 1998;23:517-21.

9. Cooney WP, Damron TA, Sim FH, Linscheid RL. En bloc resection of tumors of the distal end of the ulna. J Bone Joint Surg Am 1997;79:406-12.

10. Bieber EJ, Linscheid RL, Dobyns JH, Beckenbaugh RD. Failed distal ulna resections. J Hand Surg Am 1988;13:193-200.

11. Noble J, Arafa M. Stabilization of distal ulna after excessive Darrachs' procedure. Hand 1983;15:70-2.

12. Dingman PV. Resection of the distal end of the ulna (Darrach operation); an end result study of twenty four cases. J Bone Joint Surg Am 1952;34A:893-900.

13. Gainor BJ, Schaberg J. The rheumatoid wrist after resection of the distal ulna Jour Hand Surg 1986;10(6):837-44.

14. Hartz CR, Beckenbaugh RD Long-term results of resection of the distal ulna for post-traumatic conditions, JourTrauma 1979;19(4):219-26.

15. Gainor BJ. Lasso stabilization of the distal ulna after tumor resection: a report of two cases. J Hand Surg [Am] 1995;20:324-26.

16. Goldner JL, Hayes MG. Stabilization of the remaining ulna using one half of the extensor carpi ulnaris tendon after resection of the distal ulna. Orthop Trans 1979;3:330-31.

17. Wurapa RK, Whipple R. Distal radioulnar allograft reconstruction after giant cell tumor resection. Am J Orthop 2003; 32:397-400.

18. Stoffelen D, Lammens J, Fabry G. Resection of a periosteal osteosarcoma and reconstruction using the ilizarov technique of segmental transport. J Hand Surg 1993;18-B:144-46.

19. Hashizume H, Kawai A, Nishida K, Sasaki K, Inoue H. Ulnar buttress arthroplasty for reconstruction after resection of the distal ulna for giant cell tumour. J Hand Surg [Br] 1996;21:213-15.

20. Adams BD, Berger RA. An anatomic reconstruction of the distal radioulnar ligaments for posttraumatic distal radioulnar joint instability. J Hand Surg Am 2002;27:243-51. 